\title{
THE SHADOW-CURVES OF THE ORBIT DIAGRAM PERMEATE THE BIFURCATION DIAGRAM, TOO
}

\author{
CHIP ROSS \\ Department of Mathematics, \\ Bates College, Lewiston, ME 04240, USA \\ MEREDITH ODELL \\ 12 Meter Charters, \\ 49 Bowen's Wharf, Newport, RI 02840, USA \\ SARAH CREMER \\ Research Assistant, WestEd, \\ 400 Seaport Court, Suite 222, \\ Redwood City, CA 94063, USA
}

Received April 2, 2007; Revised February 27, 2009

The "Q-curves" $Q_{1}(c)=c, Q_{2}(c)=c^{2}+c, \ldots, Q_{n}(c)=\left(Q_{n-1}(c)\right)^{2}+c=f_{c}^{n}(0)$ have long been observed and studied as the shadowy curves which appear illusively - not explicitly drawn in the familiar orbit diagram of Myrberg's map $f_{c}(x)=x^{2}+c$. We illustrate that $Q$-curves also appear implicitly, for a different reason, in a computer-drawn bifurcation diagram of $x^{2}+c$ as well - by "bifurcation diagram" we mean the collection of all periodic points of $f_{c}$ (attracting, indifferent and repelling) - these collections form what we call " $P$-curves". We show $Q$-curves and $P$-curves intersect in one of two ways: At a superattracting periodic point on a $P$-curve, the infinite family of $Q$-curves which intersect there are all tangent to the $P$-curve. At a Misiurewicz point, no tangencies occur at these intersections; the slope of the $P$-curve is the fixed point of a linear system whose iterates give the slopes of the $Q$-curves.

We also introduce some new phenomena associated with $c \sin x$ illustrating briefly how its two different families of $Q$-curves interact with $P$-curves.

Our algorithm for finding and plotting all periodic points (up to any reasonable period) in the bifurcation diagram is reviewed in an Appendix.

Keywords: Myrberg map; orbit diagram; bifurcation diagram; attracting, repelling, and indifferent periodic point; preperiodic point; $Q$-curve; $P$-curve; Misiurewicz point.

\section{Introduction}

\subsection{Q-curves and P-curves}

Like the well-known orbit diagram of Fig. 1, a computer-generated bifurcation diagram (Fig. 2) for Myrberg's map $f_{c}(x)=x^{2}+c$ has fascinating features worthy of study. One striking feature shared by these two self-similar diagrams is the illusion of shadowy polynomial curves sweeping up and down across each of them. We call these $Q$-curves, and although they can be explicitly plotted (see Fig. 3) using Myrberg's recurrence formulas

$$
\begin{aligned}
Q_{1}(c)=c, \quad Q_{2}(c) & =c^{2}+c, \ldots, Q_{n}(c) \\
& =\left(Q_{n-1}(c)\right)^{2}+c=f_{c}^{n}(0),
\end{aligned}
$$

they are not explicitly drawn in either the orbit or the bifurcation diagram, and in fact appear implicitly in both for very different reasons. 


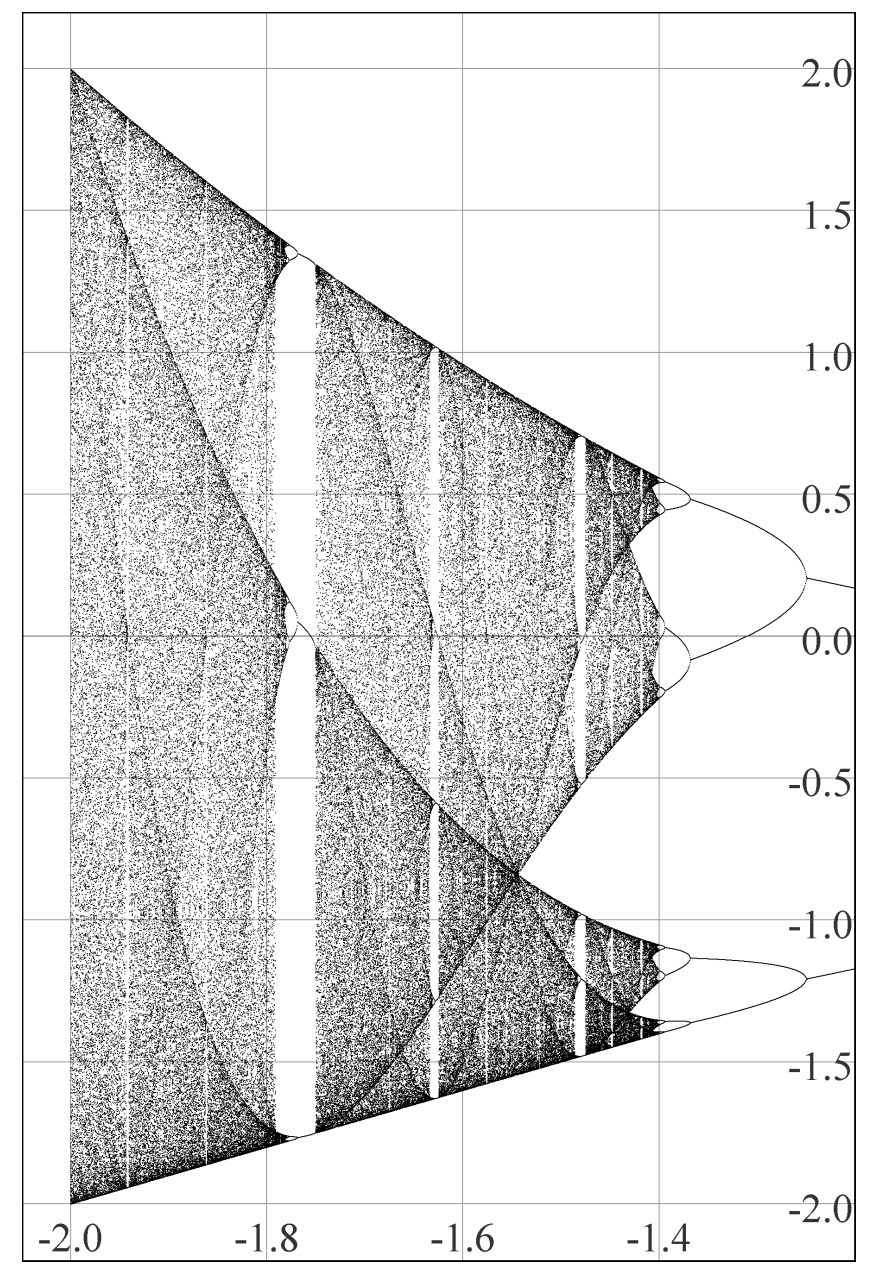

Fig. 1. Familiar Orbit diagram.

Myrberg [1963] was the first to study properties of $Q$-curves, and in particular, was interested in finding relationships among their roots and accumulations points of these roots. Using Myrberg's results, Mira [1987] further studied these accumulation points and their relationships with intersecting families of $Q$-curves. The details of why $Q$-curves can be seen in the orbit diagram, and the slopes of $Q$-curves at points where they intersect, are given in [Neidinger \& Annen, 1996]; briefly, we see $Q$-curves in Fig. 1 because chaotic orbits of 0 "pile up" more above than below $Q$-curves (or vice versa) and the abrupt difference in densities of points above versus below makes the $Q$-curves visible.

Our contribution to this topic begins with the observation that $Q$-curves also appear implicitly in a true bifurcation diagram such as in Fig. 2. This picture, drawn using our algorithm ${ }^{1}$ in

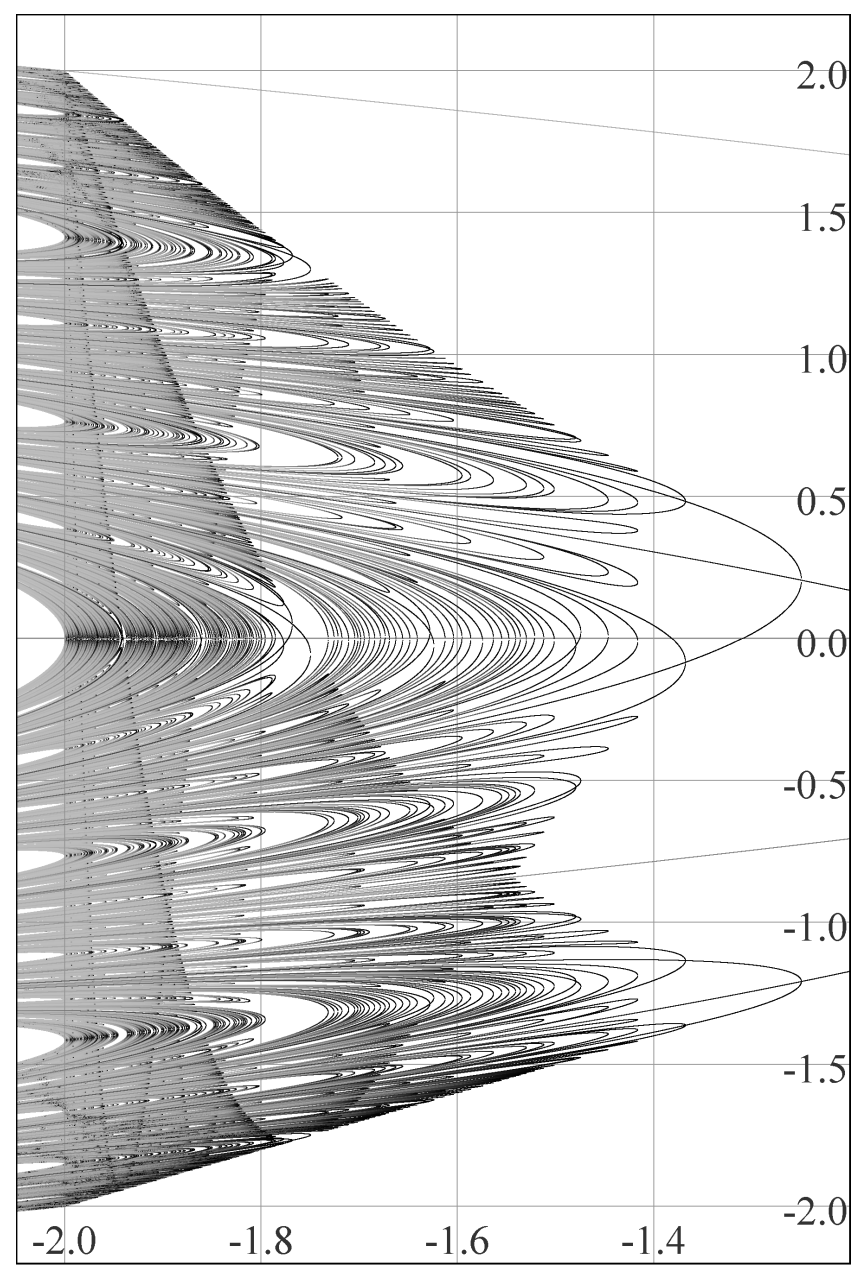

Fig. 2. Detailed Bifurcation diagram.

[Ross \& Sorensen, 2000] shows all periodic points (up through period 12 here), not just the attracting ones seen in the orbit diagram. The periodic points themselves form curves having very elongated " $\supset$ "-shapes which we shall call "P-curves". These $P$-curves all reach in from the left side of the figure, turn around (at all the various c's for which a saddle-node or period-doubling bifurcation occurs) and exit left again.

Figure 3 shows several $Q$-curves, explicitly drawn using the formulas in (1). All three Figs. 1-3 illustrate that $Q$-curves intersect one another in one of two distinctly different ways: At any such intersection, either all $Q$-curves are tangent to one another (for example, at the point $T$ in Fig. 3), or else no two are tangent (as at $M$ ). Several of each of the intersections can be seen in Fig. 3. Proving this dichotomy is a main topic in [Neidinger \& Annen,

\footnotetext{
${ }^{1}$ Briefly, the algorithm seeks crossings of iterates of $f$ and the line $y=x$. It does not use "backwards iteration", nor does it attempt solving $f_{c}^{n}(x)=x$ for $x$ in terms of $c$. A brief description of the algorithm is provided in the Appendix.
} 


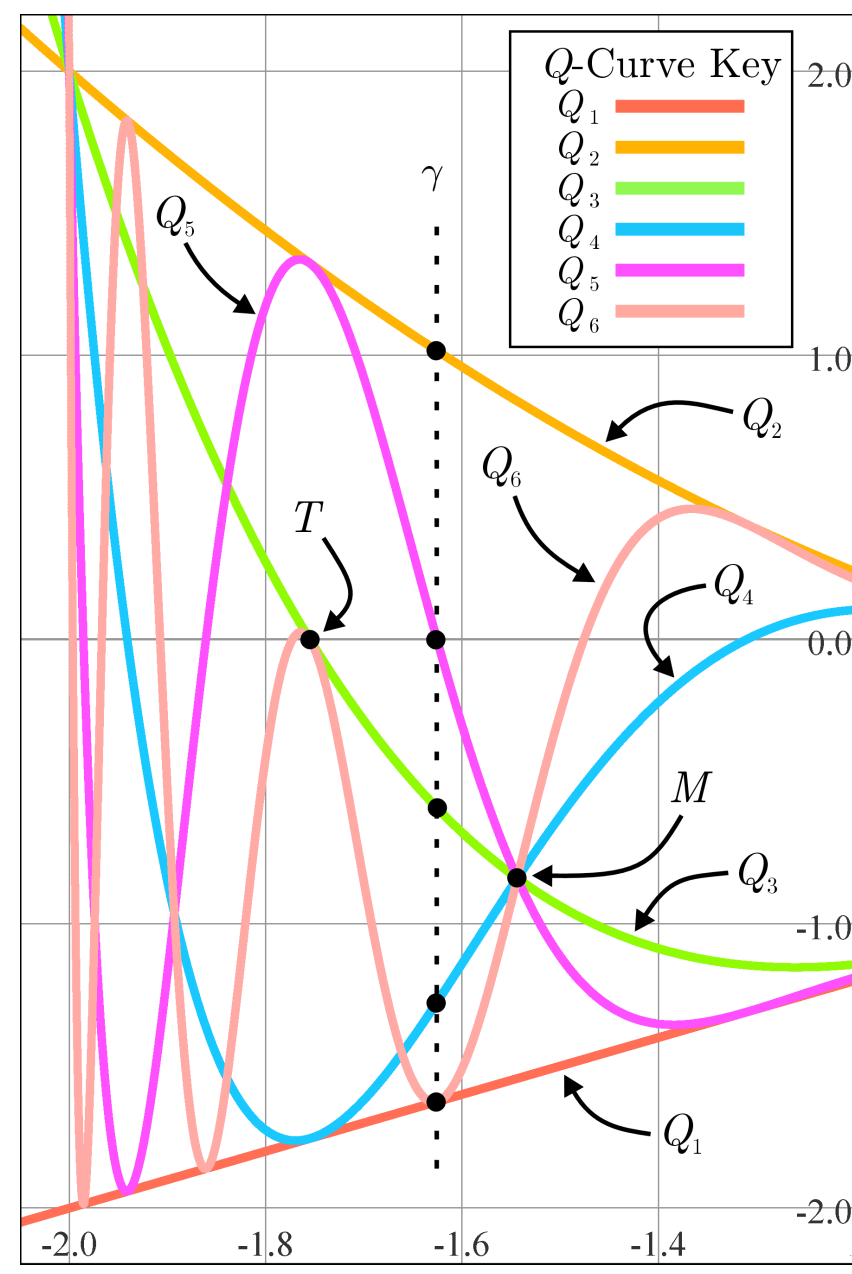

Fig. 3. The first six $Q$-curves, colored to make them easier to distinguish. We will study $Q$ - and $P$-curve interactions at $c=\gamma$. (The choice of colors is independent of the next figure.)

1996]. Our purpose is to show how this dichotomy extends to the ways the $Q$-curves interact with $P$ curves; we illustrate this in Fig. 4 as follows.

Figure 4 shows $P$-curves through prime period 6 in color and $Q_{1}$ through $Q_{8}$ in black. Now, we expect to see that a $P$-curve passes through any intersection of $Q$-curves (when such an intersection occurs at some $c$, the critical point 0 itself is either a periodic or eventually periodic point for $f_{c}$; we review this below). But we observe that at any such point where $Q$-curves are all tangent, the $P$-curve looks tangent to all the $Q$-curves. Several of these mutually tangent intersections of $P$ - and $Q$-curves can be seen (a few are marked " $t$ " and there are five along the dotted line; a close-up of the one at $*$ is offered in Fig. 8). Note such intersections are near

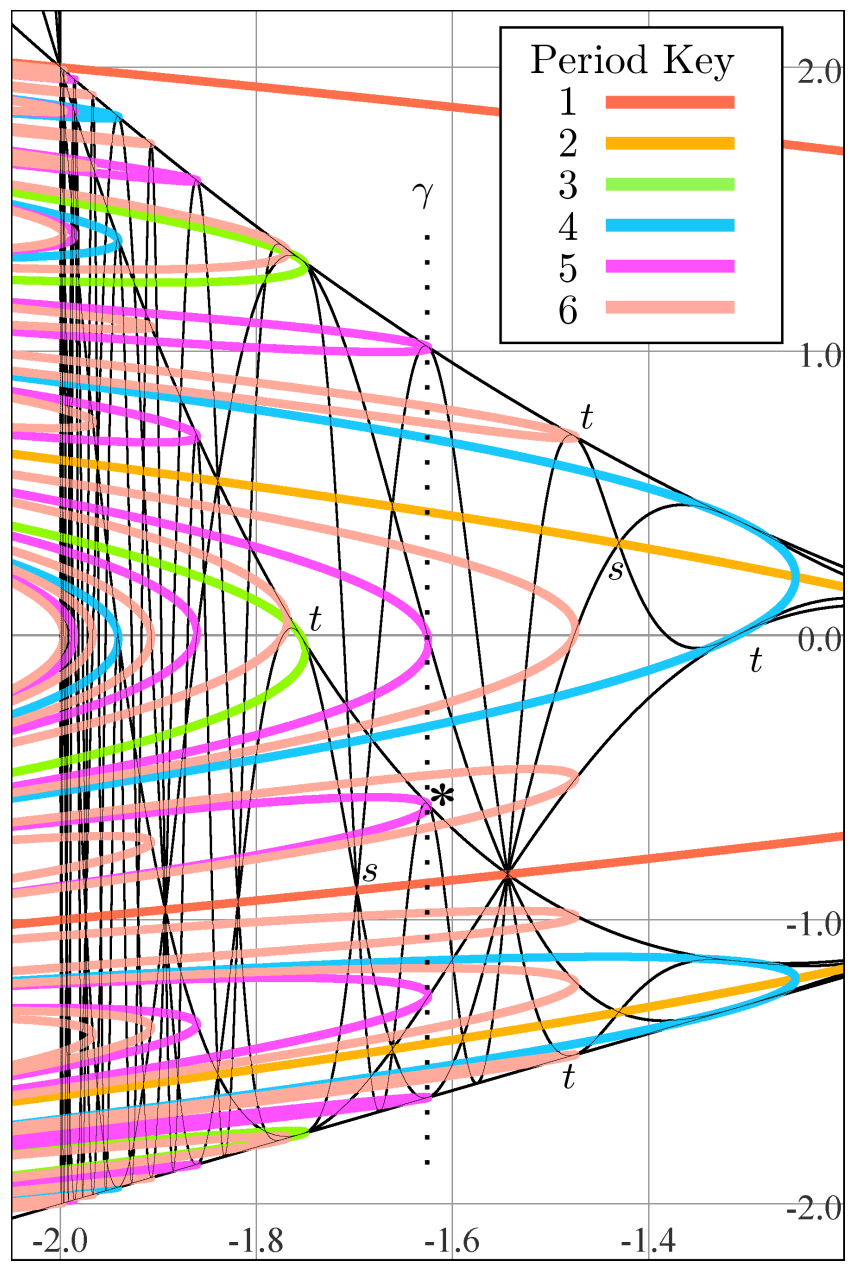

Fig. 4. $P$ - and $Q$-curve interactions. Points on $P$-curves are colored according to their prime period; $Q$-curves are black. The asterisk marks the specific $P$ - and $Q$-curve intersections studied in detail in Fig. 8.

but not at the tip of the involved $P$-curve (since for $c$ at the tip the slope of $f_{c}^{n}$ is 1 as the bifurcation occurs; after a small decrease in $c$ this slope becomes 0 and gives the $Q$-curve intersection). The $Q$-curves are lined with such intersections as the bifurcations develop from $c=0.25$ down to $c=-2$ and so become visible in Fig. 2 because of the "density difference" as there are more $P$-curves on the left than on the right of the $Q$-curves. ${ }^{2}$

A mutual tangency is one of the two distinct ways the bifurcation diagram's explicitlydrawn $P$-curves interact with $Q$-curves. The other interaction is not so obvious, but is dramatic: Wherever $Q$-curves cross and none are tangent, the corresponding $P$-curve is not tangent to any $Q$-curve there (two such crossings are labeled " $s$ "). In fact,

\footnotetext{
${ }^{2}$ The development of these bifurcations follows a specific pattern, as studied for example by Mira [1987] using his "box-withina-box" paradigm.
} 
the slope of the $P$-curve is the fixed point value of a simple linear system which when iterated starting with the slope of the lowest-numbered $Q$-curve in the intersection, produces the slopes of all remaining $Q$-curves at the intersection. (This is really what also happens in the first kind of intersection, but that simple system reduces to an even simpler constant system.) Developing the formula for these slopes is the main goal of this article.

Remark. In Fig. 4 there are intersections visible where a $P$-curve and a single $Q$-curve meet. We can always see that more $Q$-curves meet at such a point by just plotting enough of them as there are infinitely many $Q$-curves at any such intersection. However, for each additional $Q$-curve (or higherorder $P$-curve) we plot, the picture gains phenomenally more points where a $P$-curve seems to meet but one $Q$-curve.

\subsection{Orbit diagrams versus bifurcation diagrams}

The orbit diagram of Fig. 1 will be familiar to all readers; indeed it "has become the most important icon of chaos theory" [Peitgen et al., 1992]. For each $c$ in a range of $c$-values on the horizontal axis, it plots on the vertical axis the longterm behavior of the orbit of 0 for the function $f_{c}(x)=x^{2}+c$. Such long-term behavior reveals intervals of $c$ values in which $f_{c}$ has an attracting cycle and suggests chaotic behavior at other $c$ values. However, the orbit diagram is often called the "bifurcation diagram". ${ }^{3}$ But we shall follow the notation of Peitgen et al. [1992], Devaney [1989], and Strogatz [1994], and reserve that name for the diagram in Fig. 2. This genuine bifurcation diagram plots all the periodic points (up to period 12 in this particular figure) of $f_{c}$ - not just those on attracting cycles, but also those on repelling cycles; repelling cycles do not appear in the familiar orbit diagram. Note that both diagrams show perioddoubling bifurcations, as attracting cycles "split" at certain $c$-values, but the bifurcation diagram also shows how the repelling cycles persist even as $c$ decreases through such bifurcations - compare both figures in the region $c>-1.401$, for example, where $f_{c}$ undergoes the familar 1-2-4-8... period-doubling scheme. Additionally, because it shows both attracting and repelling points, the bifurcation diagram also gives a more complete picture of what happens at saddle-node bifurcations (look ahead to Fig. 7, for example). Indifferent periodic points appear in both diagrams implicitly, as they are the periodic points found right at either kind of bifurcation.

\subsection{Notation and basic definitions}

Let $f$ be one of the members of the family $f_{c}(x)=$ $x^{2}+c$ obtained by fixing a specific value of $c$. The orbit of a point $x$ under $f$ is the sequence $x, f(x)$, $f^{2}(x), f^{3}(x), \ldots$, of iterates of $x$; here $f^{n}(x)$ means the composition of $f$ with itself $n$-times. A real number $p$ is a point of period $n$ for $f$ if $f^{n}(p)=p$. If $n$ is the smallest positive integer for which this equality holds, we say $p$ has prime period $n$, and if $n=1$ we call $p$ a fixed point. If $p$ is a point of prime period $n$ for $f$, then the $n$ iterates $p, f(p), \ldots, f^{n-1}(p)$ are called the $n$-cycle to which $p$ belongs. If $\left|\left(f^{n}\right)^{\prime}(p)\right|<$ 1 , the cycle is called an attracting (or stable) cycle (and super attracting if $\left(f^{n}\right)^{\prime}(p)=0$ ); this is because one can prove the orbits of all points close to $p$ approach the orbit of $p$. The orbit diagram shows attracting cycles for $f_{c}(x)=x^{2}+c$ because if there is such a cycle for a given $c$, this cycle must attract the orbit of the critical point 0 . However the orbit diagram shows many $c$-values for which the orbit of 0 appears as a sequence of scattered points, suggesting no attracting cycle exists and instead, $f_{c}$ exhibits chaotic behavior.

Again let $p$ have prime period $n$. Now if $\left|\left(f^{n}\right)^{\prime}(p)\right|>1$, the $n$-cycle is called repelling (or unstable); in this case, there is some interval $(a, b)$ containing $p$ such that for any $x \in(a, b)$ with $x \neq p$, the orbit of $x$ moves away from the orbit of $p$. Finally, if $\left|\left(f^{n}\right)^{\prime}(p)\right|=1$, we will simply say the cycle is indifferent. In any case - no matter the value of $\left|\left(f^{n}\right)^{\prime}(p)\right|$ - the bifurcation diagram contains the point $(c, p)$.

Finally, in Fig. 4, we see two ways that $Q$-curves intersect: At any such intersection, either all $Q$ curves are tangent, or else not even a pair of them are tangent. We refer to these as intersections of the first and second kinds, respectively.

\section{Primitive Zeros and Intersections of the First Kind}

Following Myrberg, we say $r$ is a primitive zero of $Q_{n}$ if $Q_{n}(r)=0$ and $Q_{j}(r) \neq 0$ for $0<j<n$. Since

\footnotetext{
${ }^{3}$ In particular, in [Neidinger \& Annen, 1996], the orbit diagram is called the bifurcation diagram.
} 
his work may be somewhat unsung, we quickly mention some pertinent results from Myrberg [1963]. Noting that if $r$ is a specific primitive zero of $Q_{n}$, Myrberg showed that $r$ satisfies a "radical equation"

$$
r=-\sqrt{-r \underset{(1)}{ \pm} \sqrt{-r \underset{(2)}{ \pm} \cdots \underset{(n-2)}{ \pm} \sqrt{-r}}}
$$

consisting of $n-1$ radical signs and a specific "rotation sequence" of "+" and "-" signs in place of the $n-2$ " \pm " signs in (2); Myrberg's ordering law shows how the relative positions of primitive zeros can be ascertained from their respective rotation sequences. Furthermore, for those rotation sequences for which (2) admits a real solution $r$, Myrberg produced a table of all primitive zeros for $Q_{n}, 3 \leq n \leq 10$; the table has some 120 entries all calculated to 10 decimal places. (Our illustration in Sec. 2.3 considers the primitive zero $\gamma \approx-1.6254137251$ of $Q_{5}$; the rotation sequence for $\gamma$ is $(+--)$.) Myrberg developed a formula for the number of primitive zeros of $Q_{n}$, which is thus a count of the total number of saddle node and period-doubling bifurcations of period $n$ which occur in $[-2,0.25]$. Indeed, through his study of primitive zeros, Myrberg discovered the "perioddoubling" phenomena and was the first to identify Myrberg-Feigenbaum points, which are the limit points of such sequences. For example, by computing numerical values of the rightmost primitive zeros of $Q_{2^{k}}$ for $1<k \leq 9$, he determined they converged to a value between -1.40117 and -1.40115 ; today -1.401155189 is often called "the" (Myrberg) Feigenbaum point. Myrberg was aware that there are infinitely many such points in $[-2,0.25]$ for the family $x^{2}+c$. For a complete discussion of Myrberg's results and their consequences, see [Mira, 1987].

Myrberg noted that primitive zeros of $Q_{n}$ correspond to superattracting periodic points of $f$, and Neidinger and Annen [1996] discussed the slopes of $Q$-curves as they intersect at primitive zeros. Explicitly, we have:

\subsection{Intersection dichotomy part 1}

If $r$ is a primitive zero of $Q_{n}$, then 0 is on a superattracting cycle of prime period $n$ for $f_{r}(x)=x^{2}+r$. All $Q$-curves of the form $Q_{j+s n}$ meet and are tangent at the point $\left(r, f^{j}(0)\right)$, where $0<j \leq n$, for $s=0,1,2, \ldots$.
That is, let $p_{j}=f_{r}^{j}(0)$ be the $j$ th point in the periodic cycle $f_{r}(0), f_{r}^{2}(0), \ldots, f_{r}^{n}(0)=0=$ $Q_{n}(r)$. The curve $Q_{j}$ and every $n$th $Q$-curve thereafter meet and are tangent at $\left(r, p_{j}\right)$. In particular, $Q_{1}, Q_{1+n}, Q_{1+2 n}, \ldots$, all meet and are tangent at $(r, r)$, while $Q_{n}, Q_{n+n}, Q_{n+2 n}, \ldots$, all meet and are tangent at $(r, 0)$.

\subsection{Our extension of the intersection dichotomy part 1 to the slopes of P-curves}

At $r$ these $n$ families of $Q$-curves are also tangent to the corresponding $P$-curves of the bifurcation diagram which pass through those $n$ common intersections. Specifically, $P_{n, j}$ is tangent to all the $Q$-curves $Q_{j+s n}$ at $r$, where for $0<j \leq n, P_{n, j}$ is the top or bottom "half" of the $P$-curve containing the point $\left(r, p_{j}\right)$.

We remark that for any $n>3$ there are at least two separate families of $P$-curves of points of prime period $n$. Indeed Myrberg [1963] and Mira [1987] give formulas which count the number of families of $P$-curves of a given prime-period, and give laws for the order in which they appear from left to right. Fortunately, the ordering does not enter into the slope calculation. For our purposes, of the many curves which could be named $P_{n, j}, P_{n, j}$ refers to a unique curve once the specific $P$-curve is identified as the one to which the prime period $n$ point $p_{j}=f_{r}^{j}(0)$ belongs. On the other hand, for each $n$, $Q_{n}$ is a unique curve.

\subsection{Illustration}

We will prove the extension later (Sec. 3.3). Let $c=$ $\gamma \approx-1.6254$. Figure 3 shows that $Q_{1}(\gamma), \ldots, Q_{5}(\gamma)$ are all distinct and $Q_{5}$ has a root at $\gamma$, so $f_{\gamma}(0)$, $f_{\gamma}^{2}(0), f_{\gamma}^{3}(0), f_{\gamma}^{4}(0)$, and $f_{\gamma}^{5}(0)=0$ form a prime period 5-cycle for $f_{\gamma}$. In Fig. 5, the graph of $f_{\gamma}^{5}$ intersects $i d$ (the line $y=x$ ) 12 times. Now, in the boxes are two "steep" crossings representing the two fixed points for $f_{\gamma}$ as the parabola $f_{\gamma}$ also crosses $i d$ at those two points. But our concern is the ten intersections near the local max/mins; these occur in very close pairs, one of which is circled. Each pair contains one point representing one of the members of the above 5-cycle; the other five points in the pairs belong to another 5-cycle. We "zoom in" to the circled pair: In Fig. $6, G \approx(-0.6085,-0.6085)$ and $\Gamma \approx(-0.5920,-0.5920)$ are on $f_{\gamma}^{5} ;-0.5920$ is $f_{\gamma}^{3}(0)$ and -0.6085 is on the other 5 -cycle. Corresponding to these two points of prime period 5 , the 


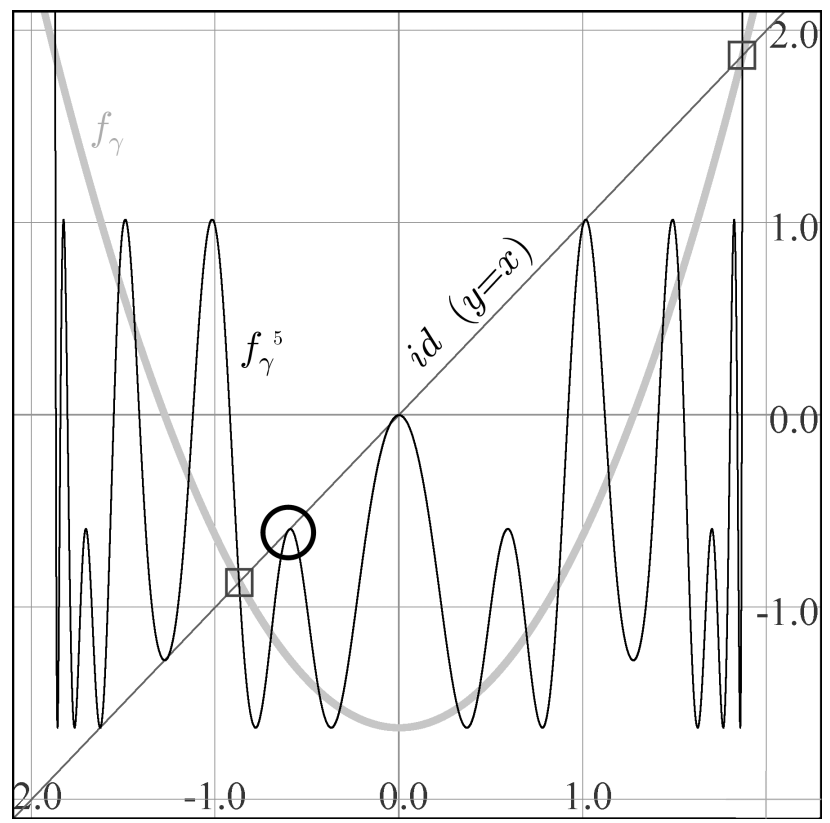

Fig. 5. There are 12 intersections of $f_{\gamma}^{5}$ with $i d$, the line $y=x$. The circled region, which contains two of the 12 , is enlarged in Fig. 6.

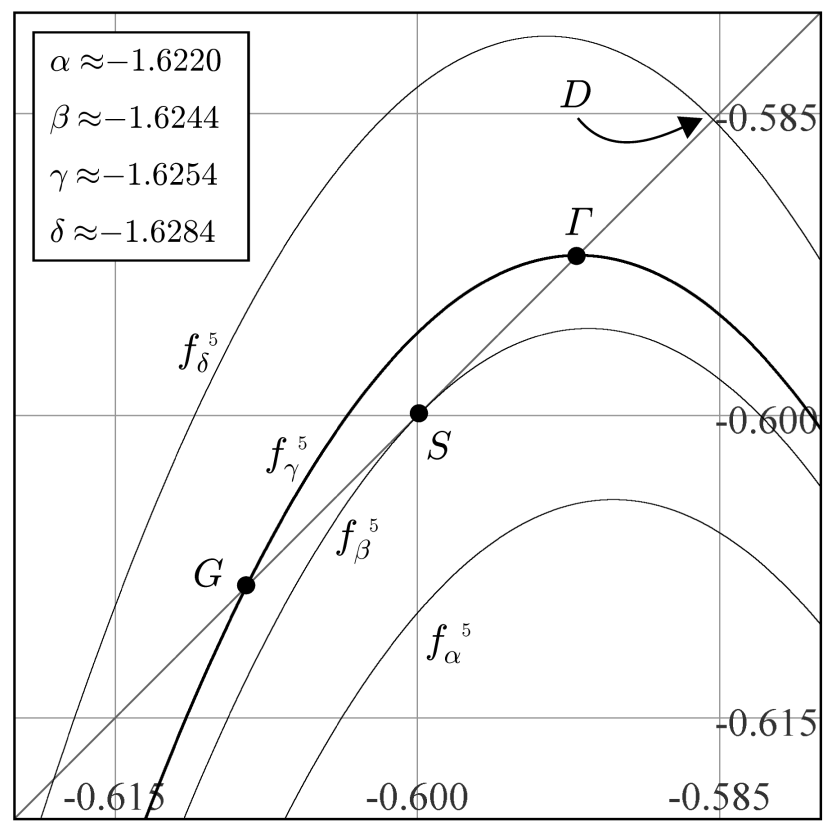

Fig. 6. Four similar curves yielding different dynamics: they show $f_{\alpha}$ has no points of prime period $5, f_{\beta}$ has a single, indifferent prime period 5 -cycle, $f_{\gamma}$ has two prime period 5 -cycles, one repelling, the other superattracting, and $f_{\delta}$ also has two, one repelling and one indifferent. At this scale, Fig. 5 would be over 17 feet wide.

portion of the bifurcation diagram shown in Fig. 7 contains $\hat{G}=(\gamma,-0.6085)$ and $\hat{\Gamma}=(\gamma,-0.5920)$. Part 1 of the intersection dichotomy and its extension say that starting with $Q_{3}$, every fifth $Q$-curve is

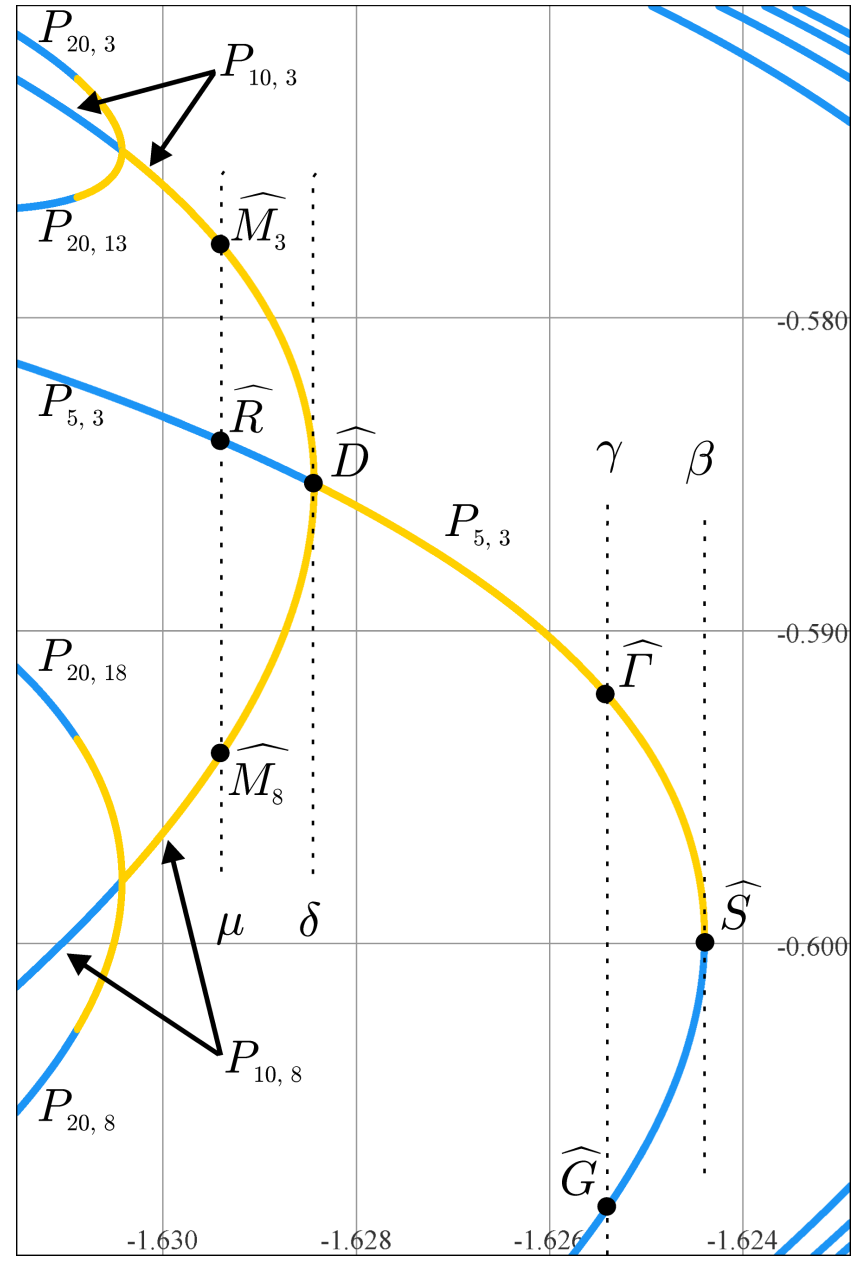

Fig. 7. Part of the bifurcation diagram, corresponding to Fig. 6, showing points of prime periods 5, 10 and 20. Points on attracting cycles are orange, repelling periodic points are in blue and indifferent periodic points are found where the two colors meet.

tangent to the $P$-curve $P_{5,3}$ at $\hat{\Gamma}$, and this is illustrated in Fig. 8 at $*$. We note in passing that $\hat{\Gamma}$ would appear on the orbit diagram, but $\hat{G}$ would not; the slope of $f_{\gamma}^{5}$ at $G$ in Fig. 6 is clearly greater than one so -0.6085 is on a repelling 5 -cycle. Also, we have focused on just one member $f_{\gamma}^{3}(0)$ of the 5 -cycle; four other pictures similar to Fig. 8 can be drawn for each of the other members of the cycle at their respective places on the bifurcation diagram. Finally, note there are some unlabeled repelling periodic points in the upper right corners of Figs. 7 and 8; from the top these are points of prime periods 18, 20, 19 and 17. Tiny portions of the bottom halves of three of these $P$-curves can be seen in the lower right corners of these figures.

In Fig. 7, note that $\hat{\Gamma}$ is near, but not at, the tip of $P_{5,3}$. The tip itself is at $\hat{S} \approx(\beta,-0.5998)$, 


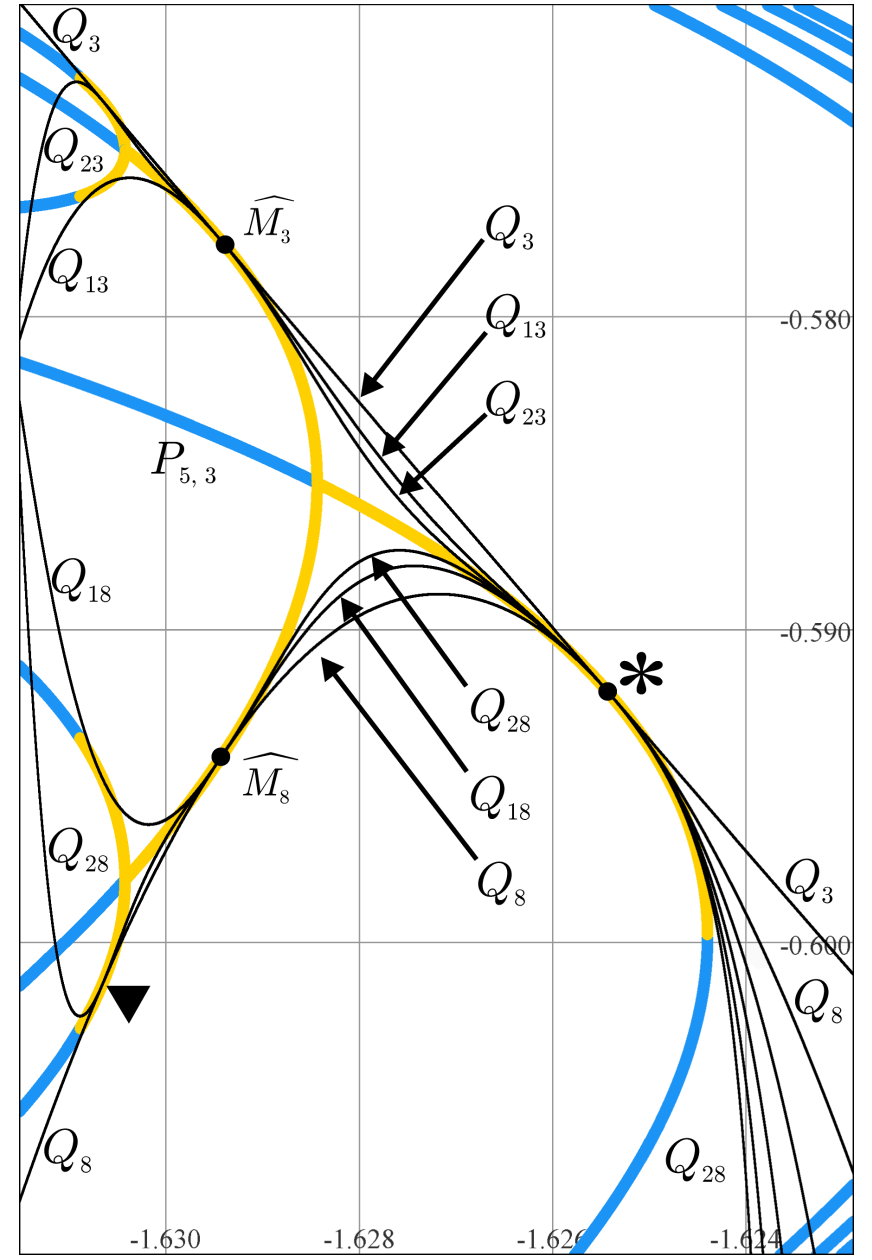

Fig. 8. $Q$-curves and the bifurcation diagram. The asterisk at $\hat{\Gamma}$ marks the same point in both Figs. 4 and 8. At this point, $Q_{3}, Q_{8}, Q_{13}, \ldots$, are all tangent to $P_{5,3}$.

where $c=\beta \approx-1.6244$ is the value at which $f_{c}^{5}$ undergoes a saddle-node bifurcation: For $c>\beta, f_{c}$ has no points of prime period 5 . Such is the case with $f_{\alpha}(\alpha \approx-1.6220)$ : in Fig. $6, f_{\alpha}^{5}$ has those five similar max/mins, but they do not cross $i d$. When $c$ decreases to $\beta, f_{\beta}^{5}$ becomes tangent to $i d$ right near each of the five local $\mathrm{max} / \mathrm{mins}$ simultaneously one of these points is $S \approx(-0.5998,-0.5998)$ in Fig. 6. Thus $f_{\beta}$ has a single prime period 5-cycle. As $c$ decreases further, $f_{c}^{5}$ crosses $i d$ twice near each of the $\max / \mathrm{mins}$, yielding two sets of prime period 5 -cycles of $f_{c}$, one repelling and the other attracting.

Importantly, only a small decrease in $c$, from $\beta$ to $\gamma$, puts one intersection in Fig. 6 of $f_{c}^{5}$ and $i d$ right at the $\max / \mathrm{min}$ itself. The corresponding 5 -cycle is superattracting - the derivative of $f_{c}^{5}$ at this intersection is 0 , and so by the chain rule, 0 itself is part of the cycle; this affirms that this $\max / \mathrm{min}$ crossing occurs when $c=\gamma$, and illustrates why $Q$-curves are tangent to $P$-curves just to the left of the tips of saddle-node bifurcations, where (after "only a small decrease in $c$ ") the superattracting periodic points are found. Figures 2 and 4 are loaded with such saddle-node bifurcations, and a family of $Q$-curves is tangent to a point near the tip of every one of them.

$P$-curves resulting from period-doubling bifurcations also abound in Fig. 2, but most are in places where the diagram is quite crowded. ${ }^{4}$ Yet two families of $Q$-curves are tangent to two points on superattracting cycles near the tip of every such $P$-curve. We will illustrate this by developing our example further. Points of prime period 5 are points of period 10. As $c$ drops below $\delta \approx-1.6284$, $f_{c}^{10}$ begins to twist around enough to cross $i d$ in two new places on each side of each point on the attracting 5-cycle. A typical period-doubling bifurcation, these ten new crossings give $f_{c}$ a single, new attracting prime period 10-cycle, while the 5-cycle becomes repelling. The intersection of $f_{\delta}^{5}$ and $i d$ at $D \approx(-0.5853,-0.5853)$ in Fig. 6 shows $f_{\delta}$ has a 5 -cycle containing -0.5853 . There the slope of $f_{\delta}^{5}$ is -1 and the changeover from attracting to repelling occurs as $c$ decreases through $\delta$. Figure 9 shows a zoom of $f_{\delta}^{10}$ also at $D$ - the period-doubling is imminent and is marked by $\hat{D} \approx(\delta,-0.5853)$ in Fig. 7. In Fig. 10 the zoom now shows $f_{c}^{10}$ after only a slight decrease in $c$ to $c=\mu \approx-1.6294$. Here the extrema are points of prime period 10 for $f_{\mu}$; again by the chain rule, 0 itself is on this

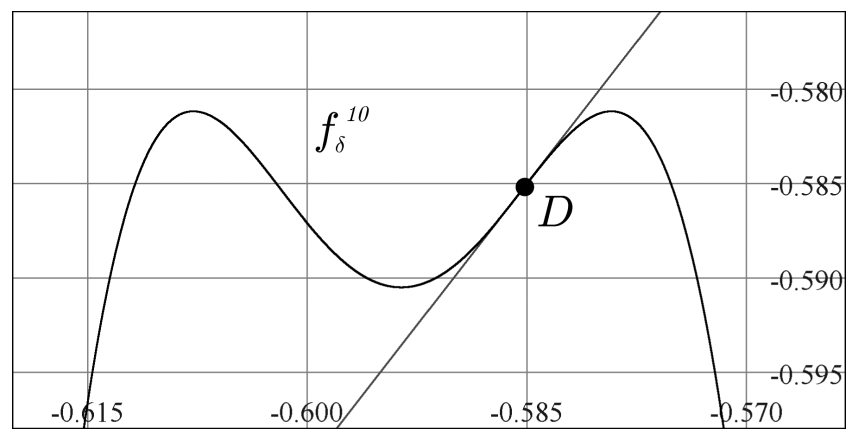

Fig. 9. A period-doubling bifurcation is imminent when $c=\delta \approx-1.6284$.

\footnotetext{
${ }^{4}$ Because it shows only prime periods from 1 to 6 , just two period doubling bifurcations occur in Fig. 4 - the prime period 2 -to-4, and the 3 -to-6. Adding more $P$-curves to see more period doubling bifurcations would crowd an already very dense picture.
} 


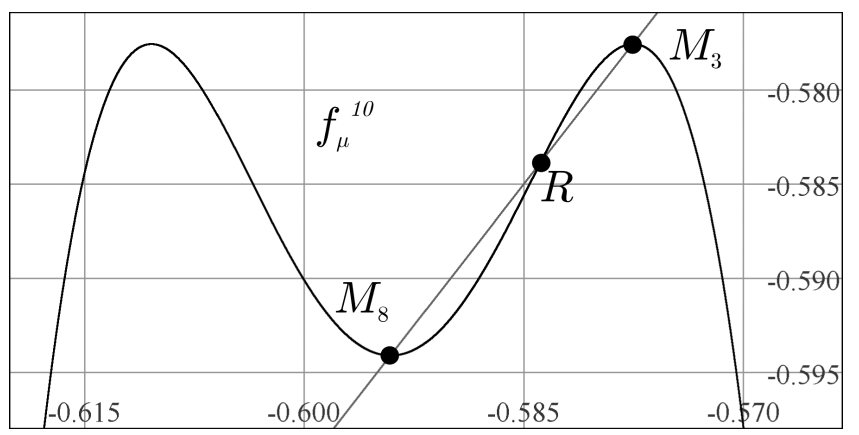

Fig. 10. Shortly after the bifurcation when $c=\mu \approx$ -1.6294 , the $\max / \operatorname{mins}$ are on the cycle, yielding a superattracting prime period 10 cycle for $f_{\mu}$.

superattracting 10 -cycle and $Q_{10}$ has a root at $\mu$. Indeed, the third and eighth iterates $f_{\mu}^{3}(0) \approx$ -0.5774 and $f_{\mu}^{8}(0) \approx-0.5941$ appear in Fig. 10 at $M_{3}$ and $M_{8}$. Correspondingly, Fig. 7 contains $\hat{M}_{3} \approx$ $(\mu,-0.5774)$ and $\hat{M}_{8} \approx(\mu,-0.5941)$. (The repelling period 5 point at $R$ in Fig. 10 also appears at $\hat{R}$ in Fig. 7.) Figure 8 shows the expected tangency of
$P_{10,3}$ and $P_{10,8}$ at $\hat{M}_{3}$ and $\hat{M}_{8}$ with the two families $Q_{3}, Q_{13}, Q_{23}, \ldots$ and $Q_{8}, Q_{18}, Q_{28}, \ldots$, respectively. The $Q$-curves thus undergo a kind of bifurcation of their own, as (from right to left) they split off from $\hat{\Gamma}$ alternately heading up or down to meet at $\hat{M}_{3}$ or $\hat{M}_{8}$. This " $Q$-curve bifurcation" happens again at $\hat{M}_{8}$, where $Q_{18}$ moves up to $P_{20,18}$, while $Q_{8}$ and $Q_{28}$ head down and are tangent again to $P_{20,8}$ at $\mathbf{\nabla}$.

\section{Intersections of the Second Kind}

If $Q$-curves meet at some $c=s$ which is not a root of any $Q$-curve, it follows that 0 is not a periodic point for $f_{s}$, but its orbit does become periodic, with prime period determined by the smallest difference between the subscripts of any pair of $Q$-curves which intersect at $s$. In this case, we say the orbit of 0 is strictly preperiodic and refer to $s$ as a Misiurewicz point.

Let $k$ and $n$ be the smallest positive integers for which $Q_{k}$ and $Q_{k+n}$ intersect at $s$. The orbit of 0 has the form

$$
\{\underbrace{0, f_{s}(0), \ldots}_{\text {no repeats }}, \underbrace{f_{s}^{k}(0), f_{s}^{k+1}(0), \ldots, f_{s}^{k+n-1}(0)}_{\text {prime period } n \text {-cycle }}, \underbrace{f_{s}^{k+n}(0), \ldots}_{n \text {-cycle }}, \underbrace{f_{s}^{k+2 n}(0), \ldots}_{n \text {-cycle }}, \ldots\}
$$

Note that $Q$-curves $Q_{1}, Q_{2}, \ldots, Q_{k-1}$, can have no intersections with any other $Q$-curves nor $P$ curves at $s$. For $0 \leq j<n-1$, let $P_{n, j}$ be the top or bottom "half" of the $P$-curve containing $\left(s, f_{s}^{k+j}(0)\right)$. From [Neidinger \& Annen, 1996] we have:

\subsection{Intersection dichotomy part 2}

Suppose that for $c=s, 0$ is strictly preperiodic, with $k$ and $n$ as above. Let $0 \leq j<n-1$. Although for any $a, b \geq 0$ the $Q$-curves $Q_{k+j+a n}$ and $Q_{k+j+b n}$ intersect at $\left(s, f_{s}^{k+j}(0)\right)$, they are never tangent, i.e. no two intersecting $Q$-curves can be tangent at such a point $s$.

Figure 4 suggests that at a Misiurewicz point, the slope of the $P$-curve is different from the slopes of the $Q$-curves meeting there. However, the slope of that $P$-curve has a fundamental relationship to the slopes of those $Q$-curves:

\subsection{Our extension of the intersection dichotomy part 2 to the slopes of P-curves}

As it passes through the intersection of the family of $Q$-curves $Q_{k+j+a n}(a=0,1,2,3, \ldots), P_{n, j}$ is tangent to none of them. At $c=s$, the slopes $D_{k+j+a n}$ of the $Q$-curves $Q_{k+j+a n}, a=0,1,2, \ldots$ are the iterates of $D_{k+j}$ for a simple linear function $y=\mathbf{m} x+\mathbf{b}_{\mathbf{j}}$. The slope of $P_{n, j}$ is the fixed point of this function.

\subsection{Proof of the tangency claims}

We prove both extensions simultaneously by analyzing the behaviors of two linear systems. Suppose $Q$-curves intersect at some $c=\bar{c}$. Let $k$ be the smallest non-negative integer, and $n$ the smallest positive integer for which $f_{\bar{c}}^{k}(0)=f_{\bar{c}}^{k+n}(0)$. If $k=0$ then $\bar{c}$ is a root of $Q_{n}$ and we are considering $Q$-curve intersections of the first kind. If $k>0$ then $\bar{c}$ is a Misiurewicz point and the intersections are of the second kind. In the arguments which follow, if $k=0$ we take $0<j \leq n$ (so that $Q_{k+j}$ cannot mean $Q_{0}$ ), whereas if $k>0$ we take $0 \leq j<n$ (so that $Q_{k+j}$ can represent $Q_{k}$ by taking $j=0$ ).

Let us write $D_{h}$ for $Q_{h}^{\prime}$, the derivative of $Q_{h}$ with respect to $c$, and $P_{n, j}^{\prime}(\bar{c})$ to mean the slope of $P_{n, j}$ at the point $\left(\bar{c}, Q_{k+j}(\bar{c})\right)$ where $P_{n, j}$ and $Q_{k+j}$ meet. 
Proposition 1. Let $k, n$ and $j$ be as above. For each $j$, the slope values $D_{k+j+a n}(\bar{c}), a=0,1,2, \ldots$ are the iterates of $D_{k+j}(\bar{c})$ for the linear function $y=\mathbf{m} x+\mathbf{b}_{\mathbf{j}}$ where $\mathbf{m}$ is the product of $2^{n}$ and the $n$ members of the prime period $n$-cycle, and $\mathbf{b}_{\mathbf{j}}$ is a combination (given in Eq. (3)) of these cycle members which depends on $j$. The fixed point of $y=\mathbf{m} x+\mathbf{b}_{\mathbf{j}}$ is the value $P_{n, j}^{\prime}(\bar{c})$.

In particular, if $k=0$ then $D_{k+j+a n}(\bar{c})=$ $P_{n, j}^{\prime}(\bar{c})=\mathbf{b}_{\mathbf{j}}$ for $a=0,1,2, \ldots$. If $k>0$ the values $D_{k+j+a n}(\bar{c})$ are pairwise unequal, and different from $P_{n, j}^{\prime}(\bar{c})$.

To prove the proposition, we first find a recursive formula for the slopes of the $Q$-curves. With
$D_{0}$ and $Q_{0}$ defined as 0 for convenience, we have $D_{h+1}=2 Q_{h} D_{h}+1$ and in general (with empty products equal to 1 ),

$$
\begin{aligned}
D_{h+t}= & 2^{t} Q_{h} Q_{h+1} \cdots Q_{h+t-1} D_{h} \\
& +2^{t-1} Q_{h+1} \cdots Q_{h+t-1}+\cdots \\
& +4 Q_{h+t-2} Q_{h+t-1}+2 Q_{h+t-1}+1 \\
= & \left(2^{t} \prod_{p=0}^{t-1} Q_{h+p}\right) D_{h}+\sum_{u=0}^{t-1} 2^{u} \prod_{p=1}^{u} Q_{h+t-p} .
\end{aligned}
$$

By Eq. (4) the slope of $Q_{k+j+a n}$ can be used to find the slope of the next higher numbered $Q$-curve $Q_{(k+j+a n)+n}$ which meets at $\left(\bar{c}, Q_{k+j}(\bar{c})\right)$ :

$$
D_{(k+j+a n)+n}=\left(2^{n} \prod_{p=0}^{n-1} Q_{(k+j+a n)+p}\right) D_{k+j+a n}+\sum_{u=0}^{n-1} 2^{u} \prod_{p=1}^{u} Q_{(k+j+a n)+n-p} .
$$

By periodicity, $Q_{h}(\bar{c})=Q_{h+a n}(\bar{c})$ for $h \geq k+j$, so when the above expression is evaluated at $\bar{c}$ we obtain

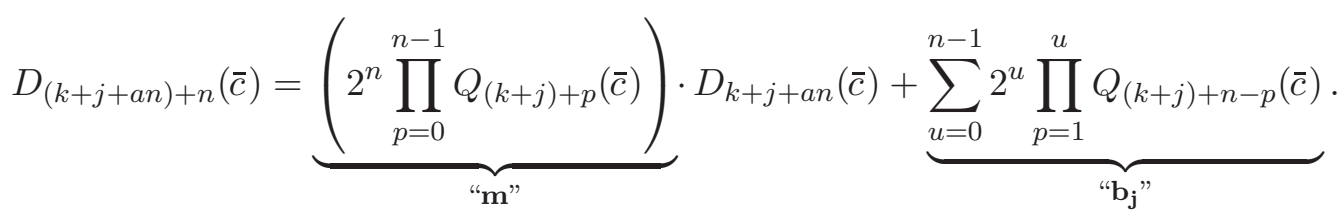

For any $j$, the product in front of $D_{k+j+a n}(\bar{c})$ is $2^{n}$ times (in some order) all $n$ iterates of the cycle, and we will call this expression $\mathbf{m}$. The summation which follows is not independent of $j$ and we will call it $\mathbf{b}_{\mathbf{j}}$. We have

$$
D_{(k+j+a n)+n}(\bar{c})=\mathbf{m} D_{k+j+a n}(\bar{c})+\mathbf{b}_{\mathbf{j}} \quad \text { for } a=0,1,2, \ldots,
$$

that is, the slopes of the $j$ th family of $Q$-curves intersecting at $\bar{c}$ are produced by iterating the function $y=\mathbf{m} x+\mathbf{b}_{\mathbf{j}}$, starting at $x=D_{k+j}(\bar{c})$.

A formula for $d x / d c$ at $\left(\bar{c}, Q_{k+j}(\bar{c})\right)$ on $P_{n, j}$ is required. We write $P_{n, j}^{\prime}$ for this derivative. In general, if the point $(c, x)$ is on $P_{n, j}$ then $f_{c}^{n}(x)=x$. Implicit differentiation of this equation with respect to $c$ and solving for $d x / d c$ yields

$$
P_{n, j}^{\prime}(c, x)=\left(\sum_{u=0}^{n-1} 2^{u} \prod_{p=1}^{u} f_{c}^{n-p}(x)\right) \div\left(1-2^{n} \prod_{p=0}^{n-1} f_{c}^{p}(x)\right) .
$$

In particular, the slope of $P_{n, j}$ at $\left(\bar{c}, Q_{k+j}(\bar{c})\right)$, that is, $P_{n, j}^{\prime}(\bar{c})$, is

$$
\frac{\sum_{u=0}^{n-1} 2^{u} \prod_{p=1}^{u} f_{\bar{c}}^{n-p}\left(f_{\bar{c}}^{k+j}(0)\right)}{1-2^{n} \prod_{p=0}^{n-1} f_{\bar{c}}^{p}\left(f_{\bar{c}}^{k+j}(0)\right)}=\frac{\sum_{u=0}^{n-1} 2^{u} \prod_{p=1}^{u} Q_{(k+j)+n-p}(\bar{c})}{1-2^{n} \prod_{p=0}^{n-1} Q_{(k+j)+p}(\bar{c})}=\frac{\mathbf{b}_{\mathbf{j}}}{1-\mathbf{m}} .
$$

If $k=0$ (the first kind of intersection) then $Q_{n}(\bar{c})=0$ is one of the factors used to define $\mathbf{m}$, so $\mathbf{m}=0$. Equation (6) becomes $D_{j+a n}(\bar{c})=\mathbf{b}_{\mathbf{j}}$ for $a \geq 1$, independently of the "first" value $D_{j}(\bar{c})$. However, the upper limit $n-1$ of the summation for $\mathbf{b}_{\mathbf{j}}$ in Eq. (5) can be replaced by $j-1$, because for $u \geq j$, the factor $p=j$ in the corresponding product is $Q_{n}(\bar{c})=0$; also in Eq. (5), $Q_{j+n-p}(\bar{c})=Q_{j-p}(\bar{c})$ because of periodicity $n$. This and Eq. (4) with 
$h=0$ and $t=j$ show directly that $D_{j}(\bar{c})=\mathbf{b}_{\mathbf{j}}$. Finally $\mathbf{m}=0$ in Eq. (7) gives $P_{n, j}^{\prime}(\bar{c})=\mathbf{b}_{\mathbf{j}}$ also. Thus for each $j, 0<j \leq n$, the $Q$-curves $Q_{j+a n}$, $a=0,1,2, \ldots$, are all tangent to the $P$-curve $P_{n, j}$ at $\bar{c}$ and their common slope is $\mathbf{b}_{\mathbf{j}}$.

If $k>0$ then because $\bar{c}$ is a Misiurewicz point, the periodic cycle 0 eventually joins is repelling [Carleson \& Gamelin, 1993]. The chain rule shows that if $p$ is any member of that cycle, then $\mathbf{m}=$ $f_{\bar{c}}^{n \prime}(p)$; because the cycle is repelling, $|\mathbf{m}|>1$. Thus the linear system $y=\mathbf{m} x+\mathbf{b}_{\mathbf{j}}$ has but one periodic point, the fixed point $\mathbf{b}_{\mathbf{j}} /(1-\mathbf{m})=P_{n, j}^{\prime}(\bar{c})$. Neidinger and Annen [1996] show that $D_{k+j}(\bar{c}) \neq$ $D_{k+j+n}(\bar{c})$, so $D_{k+j}(\bar{c})$ is not the fixed point of $y=\mathbf{m} x+\mathbf{b}_{\mathbf{j}}$ and hence its orbit is made up of distinct values, giving a concrete argument that no two $Q$-curves are tangent at $\bar{c}$, and none of them have the same slope $\mathbf{b}_{\mathbf{j}} /(1-\mathbf{m})$ as the $P$-curve through $\left(\bar{c}, Q_{k+j}(\bar{c})\right)$. In summary, the slope of $P_{n, j}$ at $\left(\bar{c}, Q_{k+j}(\bar{c})\right)$ is the fixed point of the linear system which produces the all-different slopes of the $Q$-curves through that point. The slope of $P_{n, j}$ is not equal to that of any of the $Q$-curves - in some sense $P_{n, j}$ "sits in the middle" while the $Q$-curves get steeper and steeper around it.

\subsection{Illustration}

Let $c \approx-1.83929$. Considering only two decimal places, the orbit of 0 begins $0, c, 1.54,0.54,-1.54$, $0.54,-1.54$, so that 0 joins a prime period 2 -cycle

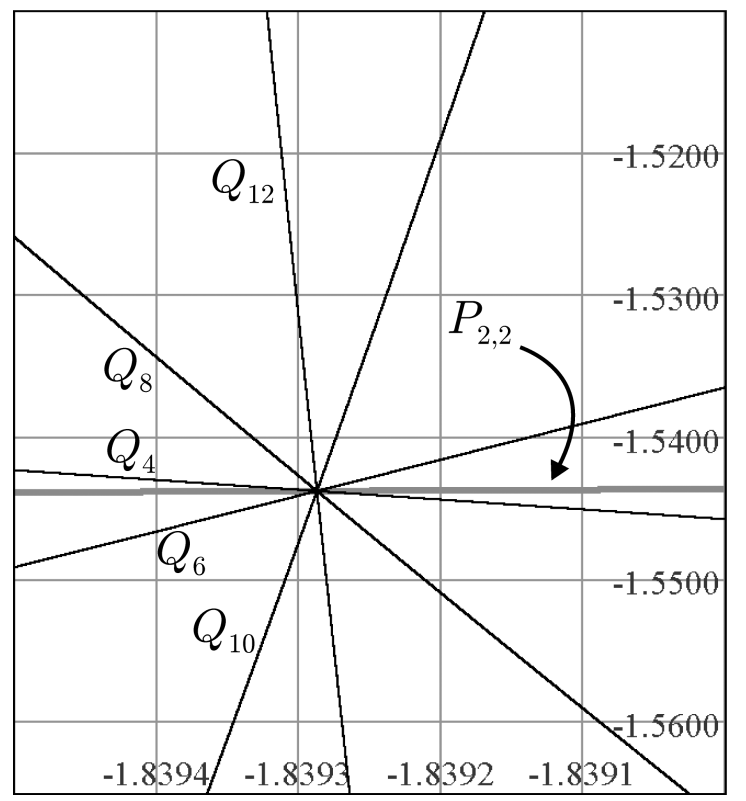

at $f_{c}^{3}(0)$; in the notation above we have $n=2$ and $k=3$. Therefore, $Q$-curves $Q_{3}, Q_{5}, Q_{7}, \ldots$, all pass through $(c, 0.5437)$, while $Q$-curves $Q_{4}, Q_{6}, Q_{8}, \ldots$, meet at $(c,-1.5437)$; this second group of curves is shown in Fig. 11. Using $D_{h+1}=2 D_{h} Q_{h}+1$, we calculated the slope of $Q_{4}$ iteratively starting with $Q_{1}^{\prime}(c)=1$. The values $\mathbf{m}=-3.3571$ and $\mathbf{b}_{1}=2.0874$ were found using Eq. (5). In Fig. 11, the ratio of the vertical to the horizontal scales is 100; the calculated values look reasonable, and in fact, they are the same (to several decimal places) as approximate values computed using $Q^{\prime}(c) \approx(Q(c+\delta)-Q(c-\delta)) / 2 \delta$, where $\delta$ was chosen quite small.

Mira [1987, Sec. 3.4] refers to Misiurewicz points as "Myrberg singular parameter values of the second type", and shows how sequences of primitive roots converge to them along the $c$-axis. In our illustration, a sequence of specific roots, one from each of $Q_{4}, Q_{6}, Q_{8}, \ldots$, is converging to $c \approx-1.83929$, alternating on the left and right of $c$ as they do so. We are not surprised then to see that when the corresponding $Q$-curves meet at $c$, the slopes become correspondingly steeper.

\subsection{Remarks}

(1) Our proofs of the dichotomies and extensions are different from and independent of [Neidinger \& Annen, 1996] with one exception: We require their

The figure to the left shows $P_{2,2}$ and the lowest numbered $Q$ curves passing through $\left(c, f_{c}^{3}(c)\right)$ where $c \approx-1.83929$. The vertical scale is exaggerated by a factor of 100 . The following table shows the slopes of these curves and which formulas were used to compute them. Here $\mathbf{m}=-3.35714$ and $\mathbf{b}_{\mathbf{1}}=2.08738$.

\begin{tabular}{ccc}
\hline Curve & Slope & Formula \\
\hline$P_{2,2}$ & 0.479070 & $\mathbf{b}_{\mathbf{1}} /(1-\mathbf{m})$ \\
$Q_{4}$ & -6.90498 & see text \\
$Q_{6}$ & 25.2684 & $\mathbf{m} Q_{4}^{\prime}+\mathbf{b}_{\mathbf{1}}$ \\
$Q_{8}$ & -82.7424 & $\mathbf{m} Q_{6}^{\prime}+\mathbf{b}_{\mathbf{1}}$ \\
$Q_{10}$ & 279.866 & $\mathbf{m} Q_{8}^{\prime}+\mathbf{b}_{\mathbf{1}}$ \\
$Q_{12}$ & -937.464 & $\mathbf{m} Q_{10}^{\prime}+\mathbf{b}_{\mathbf{1}}$ \\
\hline
\end{tabular}

Fig. 11. $Q$-curves near a Misiurewicz point on a $P$-curve. 
proof that $D_{k+j}(\bar{c}) \neq D_{k+j+n}(\bar{c})$ in the second intersection dichotomy, and their argument relies on valuation theory. Is there an easier way to show this inequality? (2) Our algorithm for drawing the bifurcation diagram can shade a periodic point according to the derivative of the underlying function when it crosses $i d$, and in particular, use darkest shades when that slope is nearest 0 . This allows us to use the fact that $Q$-curve and $P$-curve tangencies occur at superattracting points to darken the $P$-curves of Fig. 2 near their "tips" to enhance the visibility of $Q$-curves in such a tiny figure. (3) Although the tangencies occur at superattracting points, it was neccessary to plot the repelling points in Fig. 2 to visualize the $Q$-curves; the "darkened points" mentioned in the previous remark include more repelling than attracting points by a huge margin. Especially to the left of the (first) Myrberg-Feigenbaum point $\mathfrak{M} \approx-1.4012$, as the period increases the attracting points occupy much narrower and narrower intervals at the tips of the $P$-curves. If we plot only attracting points, the computer will not find many unless we "test" $c$-values at such close spacing that the plot takes an unreasonable time to complete. We illustrate this in the Appendix with two bifurcation diagrams which plot attracting points and repelling points in two colors. (4) Prime period 5 is the smallest number $n$ for which $f_{c}^{n}$ undergoes more than one saddle-node bifurcation. In fact (see Fig. 4) $f_{c}^{5}$ goes through three, so the $Q$-curves come together in groups of 5 three times across the bifurcation diagram. The intersections of $Q_{1}$ and $Q_{6}$ in Fig. 3 mark the three meetings.

\section{Two Families of $Q$-Curves in the Sine Function}

Pictures suggest the intersection dichotomies apply to bifurcation diagrams for other functions. The family $f_{c}(x)=c \sin x, c<0$ has an additional feature to consider. Figure 12 shows the bifurcation diagram for $c \sin x$ (through points of period 7 and for $c \in[-3.2,-1.2])$. Because $c \sin x$ has negative Schwarzian any attracting cycle with finite immediate basin of attraction must attract a critical point [Alligood et al., 1997]. For some values of $c$ there are two such attracting cycles, attracting the critical points $\pi / 2$ and $-\pi / 2$ respectively, thus two orbit diagrams are needed to show all the attracting cycles (see Figs. 13 and 14). Each critical point

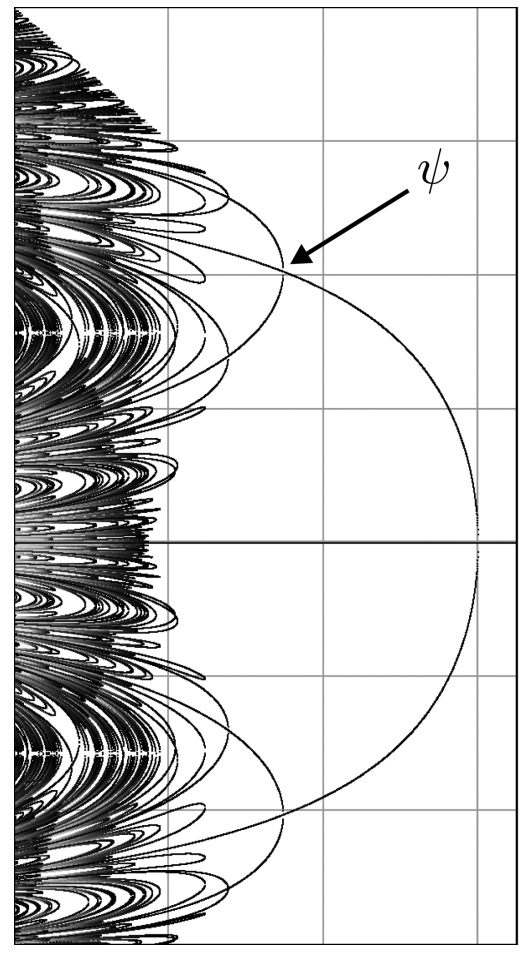

(12)

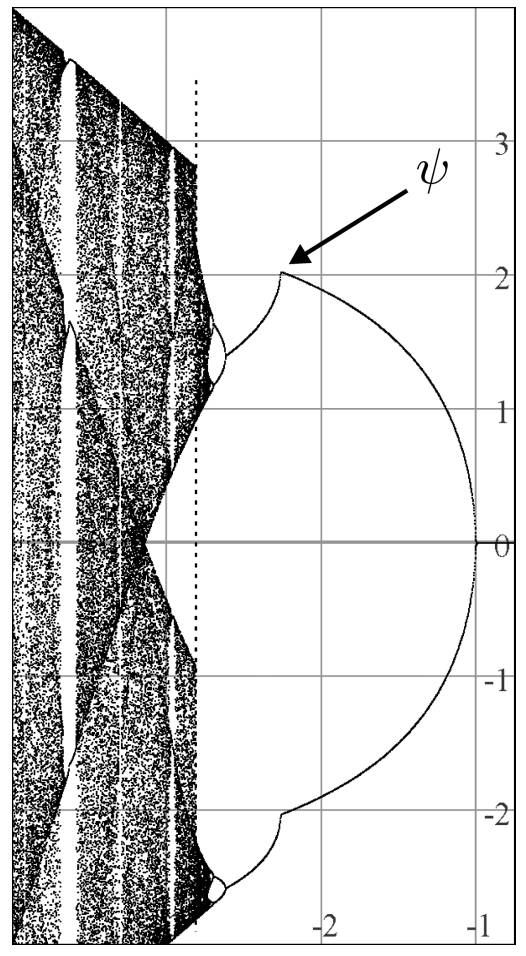

(13)

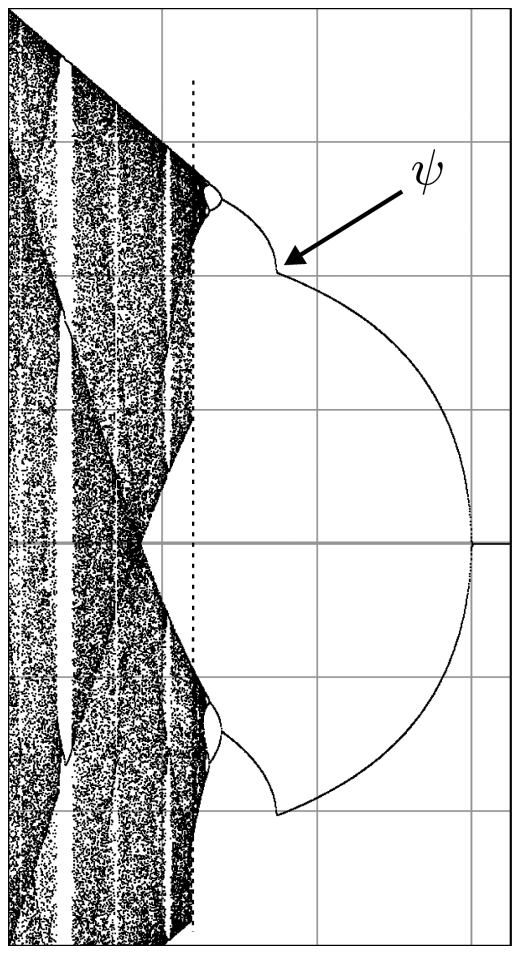

(14)

Figs. 12-14. The bifurcation diagram for $c \sin x$; the orbit diagrams for the critical points $\pi / 2$ and $-\pi / 2$ respectively. There is a pitchfork bifurcation at $\psi \approx-2.262$. 
generates a different set of $Q$-curves. Although it looks like a standard period-doubling bifurcation occurs at $\psi \approx-2.262$ in Fig. 12 , this is a pitchfork bifurcation of period 2-cycles. As $c$ decreases through this value, $f_{c}^{2}$ (not $f_{c}^{4}$ ) twists around two of its own intersections with $i d$ twice each, for a total of four new crossings resulting in two new attracting 2-cycles (see the period labeling in Fig. 15). Each new 2-cycle on the tines of the pitchfork attracts its own critical point.

Throughout all three Figs. 12-14 are two families of $Q$-like curves: Corresponding to the critical point $\pi / 2$ are $Q_{1}(c)=c, Q_{2}(c)=c \sin c$, $Q_{3}(c)=c \sin (c \sin c)$, and so on. Corresponding to $-\pi / 2$ are $K_{1}=-Q_{1}, K_{2}=-Q_{2}$, and in general $K_{i}=-Q_{i}$. Figure 15 shows a small part of the bifurcation diagram near the pitchfork bifurcation, labeling prime periods. Both the $K$ and $Q$-curves behave as expected at the familar saddle-node, period-doubling bifurcations and the Misiurewicz point $M$ in the figure. The pitchfork bifurcation presents new behavior, as it separates the $K$-curves from the $Q$-curves: In Fig. 16, all oddnumbered $K$-curves and even-numbered $Q$-curves

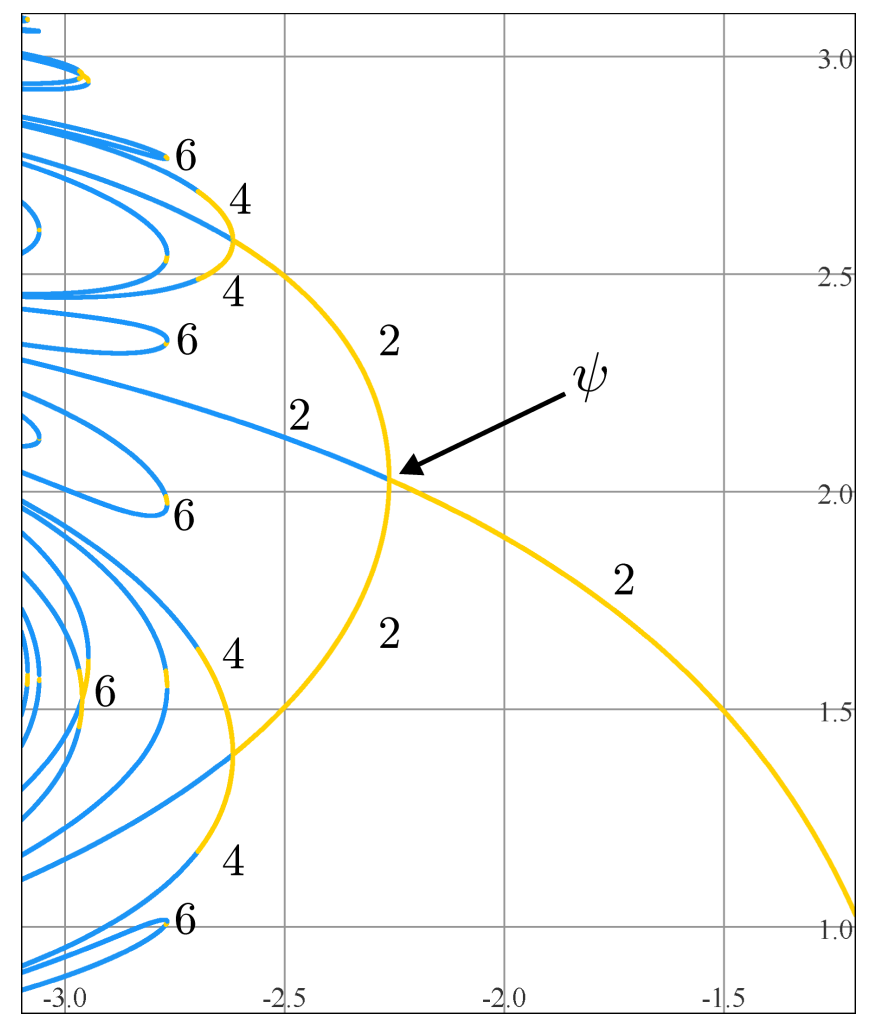

Fig. 15. A pitchfork bifurcation in $c \sin x$ at $\psi \approx-2.262$. Attracting cycles are in orange, repelling in blue. As $c$ drops below $\psi$, the attracting 2 -cycle becomes repelling, and splits into two new attracting 2-cycles.

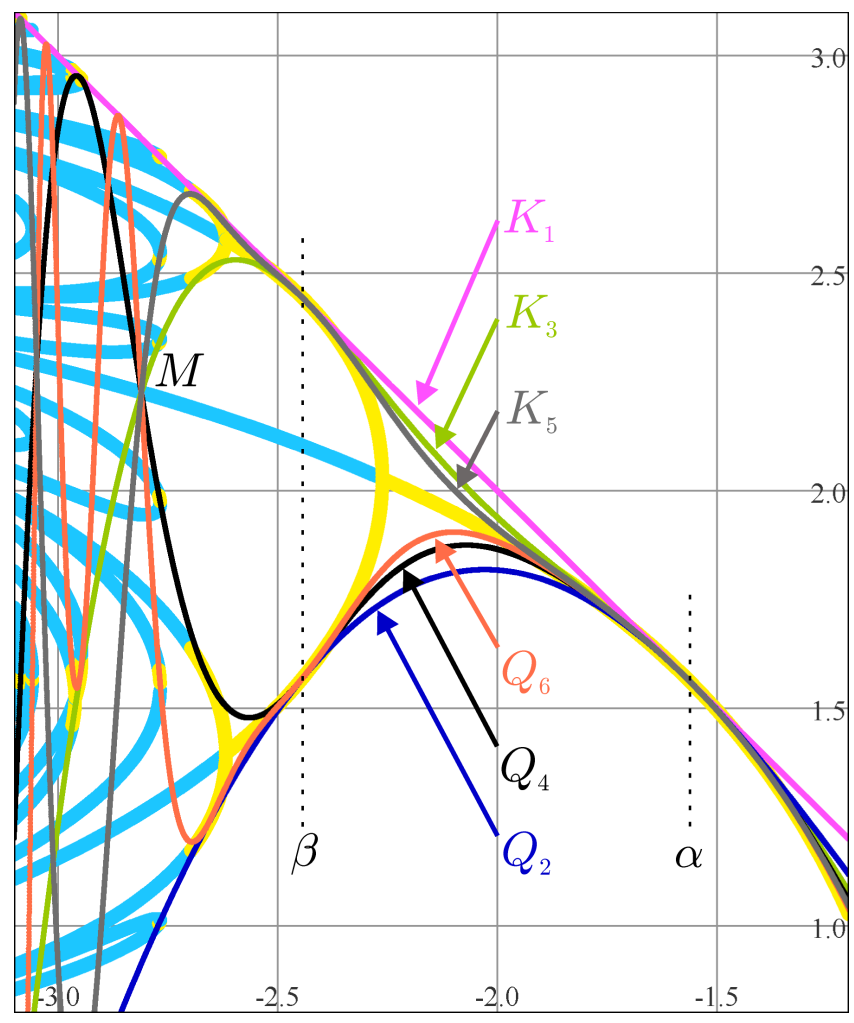

Fig. 16. Although all tangent at $c=\alpha$, the $K$-curves and $Q$ curves are separately tangent at $\beta$ on the tines of the pitchfork bifurcation. All (except $K_{1}$ and $Q_{2}$ ) come back together at the Misiurewicz point $M$.

are mutually tangent at $c=\alpha=-\pi / 2$, but at $c=\beta \approx-2.4433$ on the tines of the pitchfork, the $K$-curves are tangent to the top tine while the $Q$-curves are tangent to the bottom one. We expect this since each tine attracts its own critical point.

Also, to the right of the dotted line in Figs. 13 and 14 the vertical intervals over which the two critical points' orbits spread their chaotic behavior are different, and their corresponding sets of $K$ - or $Q$-curves envelope (in the sense of [Neidinger \& Annen, 1996]) and run through the respective regions. To the left of the dotted line, the situation is much more complicated. The Misiurewicz point $M$ in Fig. 16 plays a role here: The dotted line in Figs. 13 and 14 contains $M$ and again, marks the first $c$ value since their common intersections at $c=\alpha=-\pi / 2$ in Fig. 16 that both $K$ - and $Q$-curves meet again. To the left of $M$, it looks like both critical points $\pi / 2$ and $-\pi / 2$ spread their chaotic orbits over the same vertical intervals, at least for "most" $c$-values. But between any two such $c$-values is a thin "window" in which the above scenario is repeated on a tiny scale: A common 
attracting cycle gives way to a pitchfork bifurcation. This bifurcation separates $K$ - and $Q$-curves, the two critical points' attracting cycles and their vertical intervals of chaos for a bit, but the window closes at a Misiurewicz point where both $K$ - and $Q$-curves meet again.

\section{Conclusion}

Both the orbit diagram and the bifurcation diagram for $f_{c}(x)=x^{2}+c$ have exciting attributes to examine. The implicit appearance of $Q$-curves is one such feature they share, and in particular, the bifurcation diagram's $P$-curves of periodic points have fundamental relationships with $Q$-curves. Although the slope formulas are somewhat messy initially, they produce a simple linear system whose tame dynamics tell us about the relationships between $P$-curve and $Q$-curves. Bifurcation diagrams and $Q$-curves for other kinds of functions seem to relate in similar ways, but having more than one critical point introduces new possibilities. So, we can ask, to what extent can these interactions and our formulas be generalized?

\section{Resources}

Please visit the first author's website at www. bates.edu/ ${ }^{\sim}$ sross for additional information on bifurcation and orbit diagrams and the program used to create all the figures in this article, a copy of which can be downloaded there.

\section{References}

Alligood, K., Sauer, T. \& Yorke, J. [1997] Chaos - An Introduction to Dynamical Systems (Springer-Verlag, NY), p. 133.

Carleson, L. \& Gamelin, T. W. [1993] Complex Dynamics (Springer-Verlag, NY), p. 133.

Devaney, R. L. [1989] An Introduction to Chaotic Dynamical Systems, 2nd edition (Addison-Wesley, Reading, MA), p. 82; p. 135.

May, R. M. [1976] "Simple mathematical models with very complicated dynamics," Nature 261, 459-467.

Mira, C. [1987] Chaotic Dynamics: From the OneDimensional Endomorphism to the Two-Dimensional Diffeomorphism (World Scientific, Singapore).

Myrberg, P. J. [1958, 1959, 1963] "Iteration der Reellen Polynome Zweiten Grades," I, II, III; Ann. Academice Scientiarum Fennica, Series A, 256, p. 1-10; 268, p. $1-10 ; 336$, p. 1-18.

Neidinger, R. D. \& Annen III, R. J. [1996] "The road to chaos is filled with polynomial curves," Amer. Math. Monthly 103, 640-653.
Peitgen, H.-O., Jürgens, H. \& Saupe, D. [1992] Chaos and Fractals - New Frontiers of Science (SpringerVerlag, NY), p. 587; p. 605.

Ross, C. \& Sorensen, J. [2000] "Will the real bifurcation diagram please stand up," Coll. Math. J. 31, 2-14.

Strogatz, S. H. [1994] Nonlinear Dynamics and Chaos (Addison-Wesley, Reading, MA), p. 361.

\section{Appendix}

Readers may find useful a bifurcation diagram which distinguishes attracting and repelling periodic points, and a brief discussion of our algorithm (detailed in [Ross \& Sorensen, 2000]) used to find these points. The bifurcation diagram in Fig. 17 shows all points of prime periods 1 through 9 for $f_{c}(x)=x^{2}+c$; repelling periodic points are light blue while attracting periodic points are red and drawn thicker to make them stand out. Many of the $P$-curves are labeled, and when comparing with Fig. 4, the vertical line at $c=\gamma$ helps locate all five $P$-curves at the rightmost period-5 saddle-node bifurcation.

Briefly, our algorithm for finding periodic points of a one parameter family of continuous functions $f_{c}$ for $c$ in some interval $[L, R]$ is as follows. Divide the (horizontal) interval $[L, R]$ into subintervals of width $\triangle c$. At each $c$ which is an endpoint of one of these subintevals, in succession from $c=L$, $c=L+\Delta c, c=L+2 \Delta c, \ldots$, to $c=R$, find the periodic points $f_{c}$ has in the (vertical) interval $[B o t, T o p]$. With $c$ fixed, find points of period $n$ in $[B o t, T o p]$ by searching for crossings of $f_{c}^{n}$ and the line $y=x$ : Split [Bot,Top] into subintervals of length $\triangle v$. For each such subinterval $[a, b]=[a, a+\triangle v]$, if $f_{c}^{n}(a)-a$ and $f_{c}^{n}(b)-b$ have opposite signs then by the intermediate value theorem, $f_{c}^{n}(p)=p$ for some $p \in[a, b]$, and if $n$ is the smallest such "exponent", then $p$ is a point of prime period $n$. The quotient $m=\left(f_{c}^{n}(b)-f_{c}^{n}(a)\right) / \Delta v$ then gives a rough approximation of the slope of $f^{n}$ at the crossing; if $|m|>1$ then $p$ is declared repelling, and attracting otherwise. Plot a dot at $(c, p)$ in light blue or red accordingly.

Note that periodic points in $[a, b]$ can be missed (for example, if $f_{c}^{n}(x)-x$ changes sign twice in $[a, b]$; this can happen when $f_{c}^{n}$ is undulating fairly wildly), and the slope approximation is crude so it can happen that at the same $c$ value, some periodic points in the same cycle may get different colors although the slope of $f^{n}$ is really the same at all such points. In an effort to avoid such errors, 


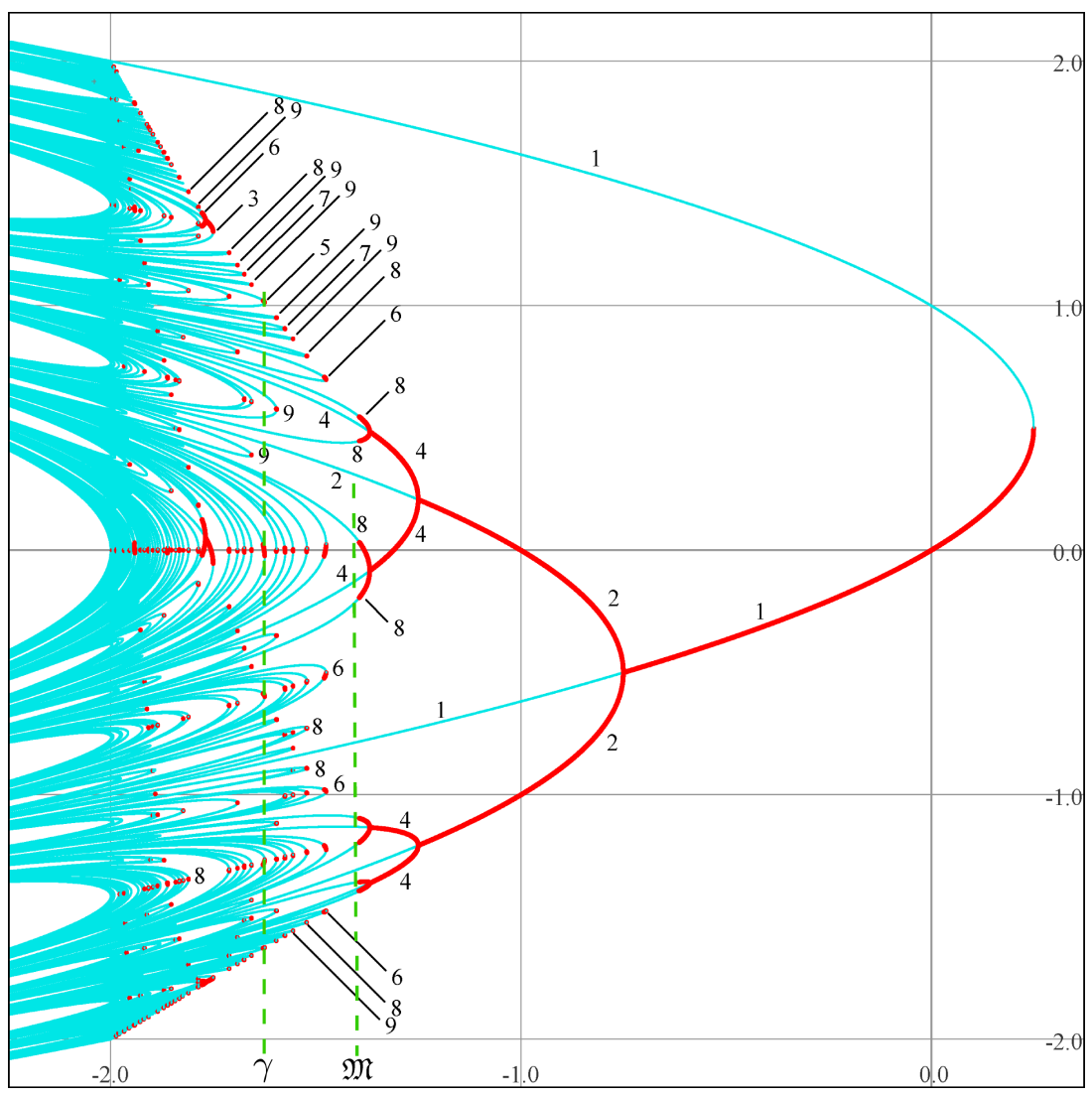

Fig. 17. Bifurcation diagram for $x^{2}+c$ showing all points of prime periods 1 through 9 . Attracting periodic points are bold red, repelling points are light blue.

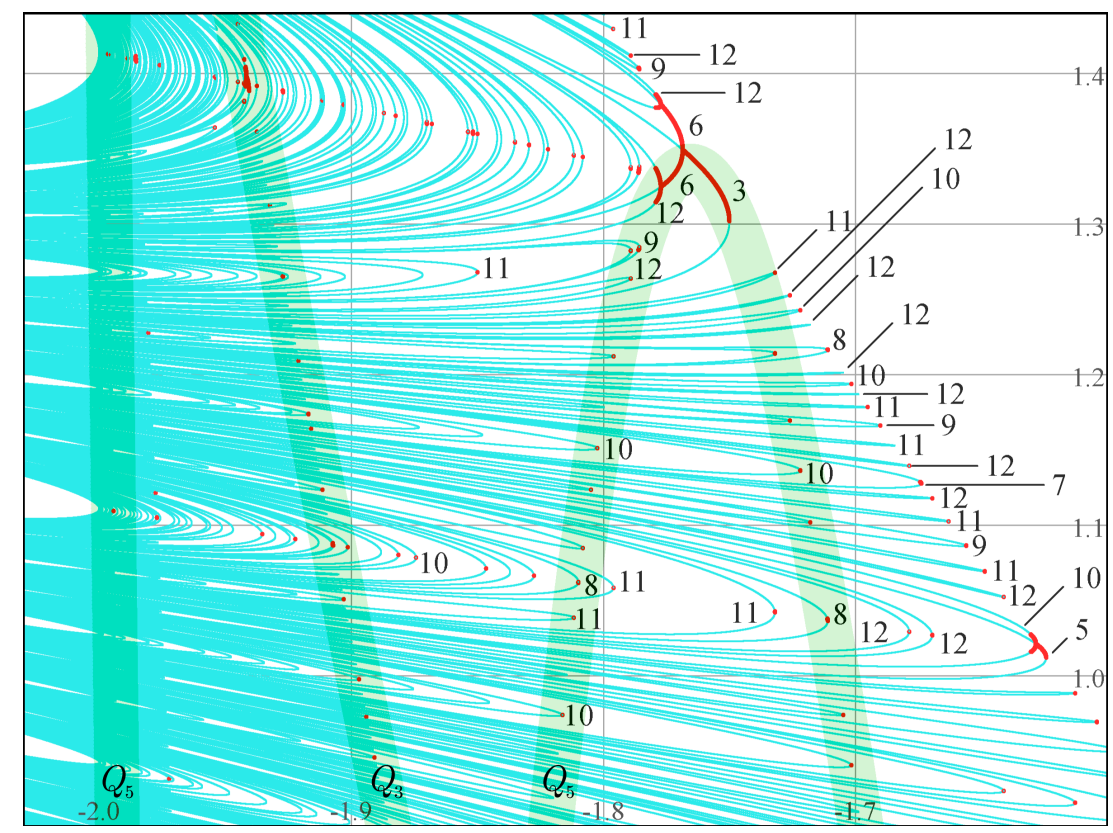

Fig. 18. A "zoom" into Fig. 17. $Q$-curves $Q_{3}$ and $Q_{5}$ are very faintly superimposed. 
$\triangle v$ can be chosen smaller at the expense of taking longer to draw the diagram.

Now, there should be an interval of red attracting periodic points at the right end of every $P$-curve in Fig. 17, and indeed the intervals are very long on the right-hand side of the diagram. However, for $c$ between -2 and the Myrberg-Feigenbaum point at $\approx-1.4012$ (marked $\mathfrak{M}$ in Fig. 17), these intervals are frequently very short, and to "help" the computer find more of them requires setting $\triangle c$ smaller. Figure 17 was drawn with $\triangle c$ set to $1 / 100$ of a pixel (and $\triangle v=1 / 10$ of a pixel), yet we see several $P$-curves that do not show any red at their right-ends. As is, this $2700 \times 2700$ pixel image took nearly 100 minutes to complete - admittedly we can get by with a much larger $\triangle c$ to the right of $\mathfrak{M}$, or to the left of -2 (where there are no attracting periodic points). Each pixel in the image represents $100 c$-values, and literally thousands of blue pixels were plotted over and over again in the effort to find just a few more red dots near the ends of $P$-curves. Note the attracting periodic points do outline $Q$-curves $Q_{1}, Q_{2}, Q_{3}$ and even $Q_{4}$ (recall however, the $Q$-curves are not tangent at the very tips of the $P$-curves but at super-attracting periodic points extremely close to those tips). Curiously, the red dots also line up on some lines that are not $Q$-curves. The reader may want to compare Fig. 17 with the hand-drawn bifurcation diagram in Fig. 4 in [May, 1976]; rotate our figure by $180^{\circ}$ first.

Finally, Fig. 18 is a "zoom" into Fig. 17, made to help show the self-similar nature of the bifurcation diagram, and again the relatively infrequent finding of attracting points despite a very small distance between sampled $c$-values. Here all points with prime periods from 1 to 12 are plotted. Many $P$-curves have been labeled and three perioddoublings (5 to 10 and 3 to 6 to 12 ) are easy to see. $Q$-curves $Q_{3}$ and $Q_{5}$ have been plotted faintly. Note that not many of the $P$-curves which line these $Q$-curves have red dots near their tips, despite the small $\triangle c$; the picture took over two hours to create and again, thousands of repelling and attracting points were plotted "unnecessarily" in our effort to find attracting points near the ends of more $P$ curves. On the other hand, coloring $P$-curves by prime period does not require so small a $\triangle c$; the $P$-curves of Fig. 4 were drawn in under a minute at an original size of $1800 \times 2700$ pixels. Finally, in shading $P$-curves according to the slopes of the corresponding crossings of $f_{c}^{n}$ and $y=x$, Fig. 2 $(1800 \times 2700$ pixels $)$ took 10 minutes. 
Copyright of International Journal of Bifurcation \& Chaos in Applied Sciences \& Engineering is the property of World Scientific Publishing Company and its content may not be copied or emailed to multiple sites or posted to a listserv without the copyright holder's express written permission. However, users may print, download, or email articles for individual use. 